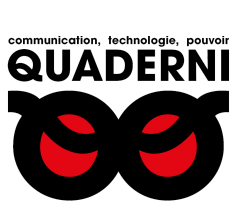

\title{
Quaderni
}

Communication, technologies, pouvoir

103 | Printemps 2021

Les ruses du hacking

\section{Romain Badouard, Les nouvelles lois du web. Modération et censure}

Pauline Brouard

\section{(2) OpenEdition}

1 Journals

\section{Édition électronique}

URL : https://journals.openedition.org/quaderni/2049

DOI : 10.4000/quaderni.2049

ISSN : 2105-2956

Éditeur

Les éditions de la Maison des sciences de l'Homme

Édition imprimée

Date de publication : 15 juin 2021

Pagination : 147-151

\section{Référence électronique}

Pauline Brouard, «Romain Badouard, Les nouvelles lois du web. Modération et censure », Quaderni [En ligne], 103 | Printemps 2021, mis en ligne le 15 juin 2021, consulté le 24 octobre 2021. URL : http:// journals.openedition.org/quaderni/2049; DOI : https://doi.org/10.4000/quaderni.2049 


\section{Romain Badouard,}

\section{Les nouvelles lois du web.}

Modération et censure

\section{Le Seuil, La République des idées, Paris, 2020}

\section{Pauline Brouard}

Sorbonne Université, GRIPIC 
Cet ouvrage aborde la thématique, qui fait couler beaucoup d'encre, de la liberté d'expression en ligne. À rebours des imaginaires libertaires constitutifs des premiers temps d'internet et du web, l'auteur apporte un regard critique sur ce qu'il désigne comme un «paradoxe démocratique », reposant sur des possibilités accrues de communication et de circulation d'information, mais surtout une concentration sans précédent des pouvoirs de contrôle et de régulation à un nombre réduit d'acteurs privés. Alliant clarté et concision, les quatre chapitres du livre brossent un tableau synthétique des enjeux législatifs, économiques, politiques et sociaux relatifs au rôle croissant des grandes entreprises numériques.

Partant du constat - corroboré par des témoignages d'usagers - de l'action de célèbres acteurs médiatiques sur la visibilité, ou inversement l'invisibilité, des contenus publiés en ligne, Romain Badouard porte à notre attention une réflexion cruciale sur les enjeux de la régulation des prises de parole. $\mathrm{Si}$ les sites web et plateformes peuvent être qualifiés d'« outils de prise de parole» (p. 12), ils impliquent néanmoins des contraintes, que ce soit en raison des formats médiatiques imposés ou de la régulation des contenus qu'ils permettent de produire, c'est-à-dire l'ensemble des processus de modération et de censure à l'œuvre sur les espaces numériques. Pour citer l'auteur, «[1]'enjeu de cet ouvrage est de dessiner des pistes pour une régulation démocratique de la parole publique en ligne, respectueuse du pluralisme du web, afin qu'internet demeure pour tous et toutes un outil au service de la liberté d'expression" (p. 19). Il semble important de préciser d'emblée le projet de l'auteur qui se donne pour horizon de formuler des pistes possibles pour la gouvernance des contenus en ligne et garantir de meilleures conditions d'expression de la parole publique sur le web.

Le premier chapitre, qui opère un retour historique sur les moments-clefs de l'histoire de la régulation d'internet, démontre la complexité de légiférer sur les conditions juridiques du débat démocratique en ligne.
La légalité des usages, par exemple, est un champ marqué par la disparité à échelle européenne et internationale. Les contrastes se situent tant du côté des textes de loi, que des conceptions mêmes de ce que doit être la liberté d'expression. L'auteur précise que les représentations culturelles déterminent en partie les cadres légaux, en comparant les actions juridiques mises en place aux ÉtatsUnis à celles qui nous sont plus familières en Europe ${ }^{1}$. Rien n'est moins simple que de savoir à qui échoit la responsabilité de la régulation des contenus ou des usages illégaux sur internet. Il convient ainsi d'opérer une distinction entre le pouvoir de régulation et la responsabilité juridique. Le chapitre permet de prendre toute la mesure de la multiplicité des acteurs en jeu : fournisseurs d'accès, hébergeurs, éditeurs, plateformes, usagers... Rappelons ici quelques étapes de la législation en France, depuis la loi de 1881 sur la liberté de la presse, jusqu'à la RGPD (Règlement Général sur la Protection des Données) en 2016, en passant par la « loi Avia » ayant pour objectif de lutter contre les contenus haineux (2020). Différentes instances prennent part à l'élaboration de ces cadres législatifs, dont des institutions publiques, mais également des acteurs du privé ou encore des émanations de la société civile comme des associations alertant sur les possibles dérives de la privatisation du marché de régulation sur internet. Comme le conclut l'auteur, «la question est moins de savoir s'il faut ou non réguler les contenus en ligne que d'identifier qui est légitime pour le faire» (p. 43).

Le deuxième chapitre traite des enjeux de modération, de filtrage et de censure des plateformes. Romain Badouard relève «quatre piliers » des pratiques de modération en s'appuyant sur celles mises en place sur Facebook et Youtube. Premièrement, il s'agit

1. Notons ici que l'Europe, dans l'ensemble de cet ouvrage, est plutôt entendue au sens des pays appartenant à l'Union européenne. Les données citées par l'auteur concernent majoritairement la France, l'Allemagne et le Royaume-Uni, même si d'autres exemples d'autres pays européens sont mentionnés. 
de l'édiction de « règles communes (...) qui définissent les conditions de prise de parole " et qui précisent les contenus non-autorisés. Deuxièmement, il est fait mention d'" un double mécanisme de signalement participatif et d'évaluation externe", puis troisièmement, des ressorts algorithmiques de modération, définis comme des "dispositifs techniques de modération automatique». Le quatrième point concerne les discours des plateformes sur leurs propres pratiques de modération (p. 48). Le chapitre explique les rouages de cette régulation, en insistant sur l'opacité des pratiques de modération et en convoquant les travaux d'autres chercheurs, tels que ceux d'Antonio Casilli sur le digital labor $^{2}$ ou ceux de Benjamin Loveluck sur le «vigilantisme numérique $^{3}$. " Ce chapitre souligne la double dimension - à la fois technique et sociale - de la modération et des dérives censoriales qui peuvent en découler. Plusieurs études relativisent l'efficacité de la modération automatique en raison non seulement des pratiques de contournement des usagers, mais aussi de la binarité du raisonnement algorithmique qui peut se résumer de la sorte : « mot interdit ou autorisé, présence de nudité ou non, atteinte au droit d'auteur ou présentation d'une licence d'exploitation " (p. 55). La complémentarité d'une modération automatique et de pratiques de signalement par les usagers eux-mêmes s'impose, en dépit des limites attestées de ce type de «censure participative " pouvant mener à un étouffement de certaines prises de parole. Romain Badouard conclut ce chapitre sur les processus d'invisibilisation des discours comme forme de régulation alternative à la suppression de contenus. Il ne s'agit plus de «bloquer un discours, mais plutôt d'en paramétrer le public, afin de contrôler au maximum le processus de réception» (p. 60). Tout en mentionnant des initiatives prometteuses de régulation publicitaire,

2. Antonio Casilli, En attendant les robots. Enquête sut le travail du clic, Paris, Seuil, 2019.

3. Benjamin Loveluck, «Le vigilantisme numérique, entre dénonciation et sanction. Auto-justice en ligne et agencements de la visibilité », Politix, n 115, 2016. l'auteur appelle de ses voux une implication active des pouvoirs publics sans en préciser la teneur.

Le troisième chapitre s'intéresse au rôle de médiation des plateformes numériques, et de leurs enjeux politiques et économiques. Cet avant-dernier chapitre s'ouvre sur les scandales ayant éclaté à propos du marché publicitaire sur internet, ainsi que du financement (direct ou indirect) de sites promulguant des discours haineux ou complotistes. Ces révélations ont eu pour écho de mettre en lumière la question de la régulation des contenus. Pour éviter les allégations de censure ou d'atteintes à la liberté d'expression, les plateformes ont mis en place deux stratégies distinctes, d'une part expliciter leur rôle d'intermédiaire et leurs actions de régulation et de modération, d'autre part réduire la visibilité de certains contenus. Si ce chapitre éclaire certains aspects du marché économique que constitue l'information, plusieurs zones grises restent en suspens. Par exemple, l'absence d'une politique harmonisée de régulation ou les contradictions inhérentes au "pluralisme des normes en vigueur sur internet $»(p .85)$ sont autant de sujets ouvrant à de passionnantes pistes de recherche, mais qui ne sont qu'ébauchées dans ce court ouvrage.

Le quatrième chapitre part d'exemples de mobilisations d'usagers pour lutter contre les pratiques de régulation abusive. Insistons sur le cas, développé ici, de l'impossibilité de publier des photographies ou images où apparaissent des «tétons féminins » quand celles de «tétons masculins » circulent librement. Cette mobilisation est devenue paradigmatique de la lutte contre les censures discriminatoires. L'auteur illustre ainsi la façon dont la société civile peut participer activement à la construction de normes de régulation. Celles-ci influencent des écarts de procédure de modération et renvoient également à « $l a$ question de la représentation des publics dans la gouvernance des plateformes » (p. 91). Pour sortir de la dichotomie qui oppose censure et non-régulation ou laxisme, l'auteur présente plusieurs modèles alternatifs de gestion, que l'on peut synthétiser avec les quatre proposi- 
tions ci-dessous.

1. D'abord, la responsabilisation des usagers, en partant de l'établissement de règles communes ou de charte. C'est le cas de la plateforme Reddit qui propose une " Reddiquette » édictant des normes de bonnes pratiques, pouvant être appliquées à tous les forums sans obligation d'application, ce qui n'est pas sans soulever des réprobations.

2. Puis, la proposition de déléguer la fonction de régulation aux usagers, dont la forme, tout en ayant l'avantage de permettre la personnalisation par l'usager lui-même de ce qu'il voit sur la plateforme, "porte en elle un certain nombre de dérives dont les pratiques de censure collective ou de justice punitive constituent deux exemples symptomatiques " (p. 94). Les pratiques de fact-checking - soit performées directement par des individus ou des groupes d'usagers, soit confiées à des organismes spécialisés - interpellent sur les conditions concrètes de cette forme de régulation. Ces collaborations sont actuellement à un stade expérimental et il est difficile d'en évaluer les conséquences concernant la liberté d'expression sur les plateformes.

3. Ensuite, une "stratégie du contrediscours ", spécifique de certaines luttes contre les fake news ou les discours haineux, qui consiste à s'opposer par le dialogue aux stratégies discursives alimentant les conflits de manière agressive et haineuse. L'enjeu est "moins de chercher à convaincre les internautes en question qu'à occuper l'espace du débat pour délégitimer les propos racistes, sexistes ou homophobes aux yeux de la majorité silencieuse qui consulte les échanges sans $y$ prendre part » (p. 98). Tout en nuançant les possibilités d'en évaluer les retombées, l'auteur souligne que les plateformes y portent attention, ne serait-ce que pour potentiellement « les intégrer directement à leurs propres dispositifs de lutte contre la désinformation et les discours de haine" (p. 100). Reste encore et toujours la question des modalités de l'intégration de ces "organisations partenaires " (p. 100) et des effets que cela aurait sur leurs actions elles-mêmes, puisqu'elles seraient dès lors soumises aux injonctions des acteurs pri- vés, ce qui porterait atteinte à leur indépendance et à leur prise de partie dans le débat public.

4. Enfin, la dernière alternative concerne les engagements de « communautés techniques » pour promouvoir les libertés numériques et des conditions d'expression démocratique en ligne (p. 103). En réponse à la centralisation des pouvoirs de contrôle sur internet, certaines communautés promeuvent des valeurs liées au respect du droit humain et la liberté d'expression. Mentionnons l'initiative, sous l'impulsion de la World Wide Web Foundation (dont l'un des créateurs n'est d'autre que Tim Berners-Lee, l'inventeur du web), d'un « "contrat pour le web", qui expose neuf principes fondamentaux que les signataires s'engagent à respecter pour conserver un web ouvert, décentralisé et interopérable " (p. 103).

Au bilan, ce chapitre lance des pistes sur lesquelles l'auteur semble prendre le soin de ne pas trop s'aventurer, tant la tension entre « liberté d'expression " et "régulation " semble mener à une situation inextricable qui alimente bien des controverses sur la question de la régulation de contenu et de la liberté d'expression.

Romain Badouard achève l'ouvrage sur trois principes permettant de penser la gouvernance politique et démocratique sur internet : celui d'une transparence des procédures de modération des grandes entreprises numériques, impliquant explication et justification des prises de décision ; celui d'une reconnaissance d'un droit d'appel, en lien avec le premier principe, qui stipule que l'on sache pourquoi une publication a été soumise à de la modération, ainsi que la possibilité de pouvoir contester et discuter la décision, puis éventuellement rétablir la publication «selon les conditions initiales » (p. 107) ; et celui de la création d'un organisme gestionnaire sous la forme d'une "agence publique et indépendante, qui aurait pour fonction de contrôler l'action des plateformes en matière de régulation des contenus" (p. 107). L'auteur insiste sur la reconnaissance de nouveaux droits et de nouvelles régulations qu'il reste à inventer. 
Si ce triptyque de principe ne se révèle être qu'une esquisse de solution, la conclusion atteste de la complexité des questions législatives du marché de l'information et de la richesse des pistes à explorer. 